\title{
BED BUG BITES ON ATHLETES
}

\author{
V. Valtchev ${ }^{1 *}$, M. Chalakov ${ }^{2}$ \\ ${ }^{1}$ Department of Physiology and Biochemistry, National Sports Academy “Vassil Levski”, Sofia, \\ Bulgaria \\ ${ }^{2}$ Department Language Learning and Information Technology, National Sports Academy \\ "Vassil Levski", Sofia, Bulgaria
}

\begin{abstract}
PURPOSE: Determine the number of athletes bitten by bed bugs and the location of the bite. METHODOLOGY: The diagnosed cases based on clinical anamnesis and examination by a dermatologist. RESULTS: 80 athletes ( 25 women and $55 \mathrm{men}$ ), aged 21 to 24, were investigated. All of them were complaining of itching and rash. After the clinical anamnestic information was collected and the dermatological examination performed, rashes because of bed bug bites were found in 55 of the subjects studied. The diagnosed lesions were linearly or annularly grouped and some of them have had a characteristic hemorrhagic center at the location of the bite. CONCLUSION: There is very little information on the frequency of bed bug bites in athletes in the available literature. This aroused our interest to present this material, which based on registered bed bug bites in athletes specializing in football. Bed bug bites and the accompanying skin irritation, which leads to unpleasant itching, impair the quality of human life.
\end{abstract}

Key words: sports, bed bugs, athletes.

\section{INTRODUCTION}

In recent years, there has been an increase in the number of bed bug bites worldwide. One possible explanation for this is frequent travel to different countries. Participation in the world and European competitions, as well as staying in international sports camps is a prerequisite for the migration of bed bugs.

Promiscuity (indiscriminate sex), immigration of people and the sale of second-hand furniture, and books also contribute to their spread. Bed bugs were most commonly found in hotels, dormitories, sports camps, locker rooms, social homes, cinemas and theaters, clothing stores, and office buildings - places where residents change frequently (1-3).

Bed bugs have existed since ancient times. 3,500 years old bed bugs found in tombs in Akhenaten (Egypt). The causes of large epidemics of bed bugs are poor hygiene and

\footnotetext{
*Correspondence to: Valentin ValtchevDepartment of Physiology and Biochemistry, National Sports Academy "Vassil Levski", Acad. Stefan Mladenov' Street, Sofia, Bulgaria , E-mail: drviv@abv.bg
}

the concentration of many people (e.g., sports camps). At the beginning of the 20th century, nearly a third of London's population is affected. After World War II, the frequency of bites from bed bugs decreased in developed countries, because the newly discovered insecticides like DDT (dichlorodiphenyltrichloroethane) are most effective in controlling bed bugs (1-3).

Worldwide, reports of the spread of the bed bugs Cimex lectularius and C. hemipterus (in Australia) are increasing. Irrespective of hygiene conditions, the insects can be especially found in hotels and hostels as well as in other holiday accommodation, health facilities, transport and in the secondhand trade. From these localities, the insects spread to private households. A bed bug infestation may well have health consequences and constitutes a heavy burden for those affected. The global spread of bed bugs appears to be multifactorially conditioned and the causes are controversially discussed (4).

Although in the second half of the 20th century C. lectularius has been rather scarce in Europe, a dramatic increase of these parasites was 
noted during the past years, particularly in the UK and the USA. This increase is thought to be related to the higher incidence of international travel and migration (5).

Travelers are at particular risk for infestation, as bed bugs have been detected in aircraft, boats, trains, and hotels. The global bed bug population is estimated to be increasing by 100-500 \% annually. In one nationwide survey, $99.6 \%$ of United States (U.S.) pest professionals reported that they have treated bed bugs in the past year, and $68 \%$ of U.S. pest professionals reported that bed bugs are the most challenging pest to treat (6).

Bed bug bites occur in places such as the neck, arms, hands or other parts of the body during sleep, causing a painful rash that can lead to itching, loss of sleep and discomfort in the victims. In athletes, bed bug bites can lead to a decrease in their fitness due to severe itching (7).

The aim of the study is to determine the number of athletes bitten by bed bugs and the location of the bite.

\section{METHODOLOGY}

Contingent on the study: 80 athletes (25 women and $55 \mathrm{men}$ ), aged 21 to 24 , were investigated. All of them were complaining of itching and rash. The conducted study was for a one-year period. All subjects are majoring in football at NSA "Vasil Levski". All of them
VALTCHEV V., et al. have participated in preparatory sports camps in the country and abroad, compared to their year-round sports training, the training cycles, consistent with the previously prepared preparatory plan by the teams in which they compete. Bedbug bites in all athletes occurred at night. All were treated orally with Desloratadine $5 \mathrm{mg}$ tabl. for 30 days and a topical corticosteroid (Methylprednisolone aceponate $0.1 \square$ cr. twice daily for 14 days) with very good results.

Methods: The diagnosis was based on clinical anamnesis and examination by a dermatologist.

\section{RESULTS AND ANALYSIS}

After collecting medical history and dermatological examination of 80 athletes with complaints of itching and rash, it has been found that 55 of them have rashes due to bed bug bites (Figure 1). The diagnosed lesions are grouped linearly or annularly and some of them have a characteristic hemorrhagic center at the site of the bite. The itching is strongest in the morning.

Cases of bed bug bites are more common in men - 41 (82\%), compared to women -14 (28\%) (Figure 2).

Localization of the rashes caused by bugs shows that $20(36 \%)$ of the athletes are bitten on the torso and $11(20 \%)$ - on the upper and lower limbs and $24(44 \%)$ - on the torso + upper and lower extremities (Figure 3).

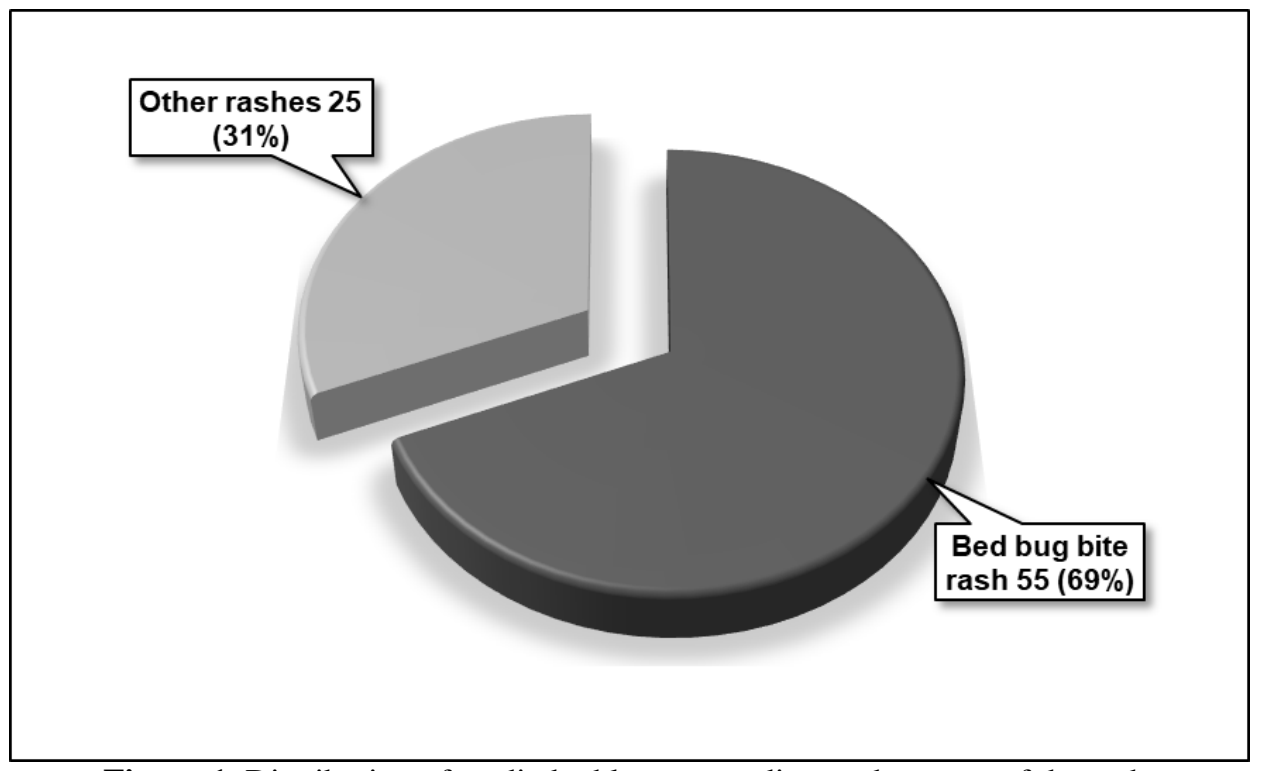

Figure 1. Distribution of studied athletes according to the cause of the rash. 


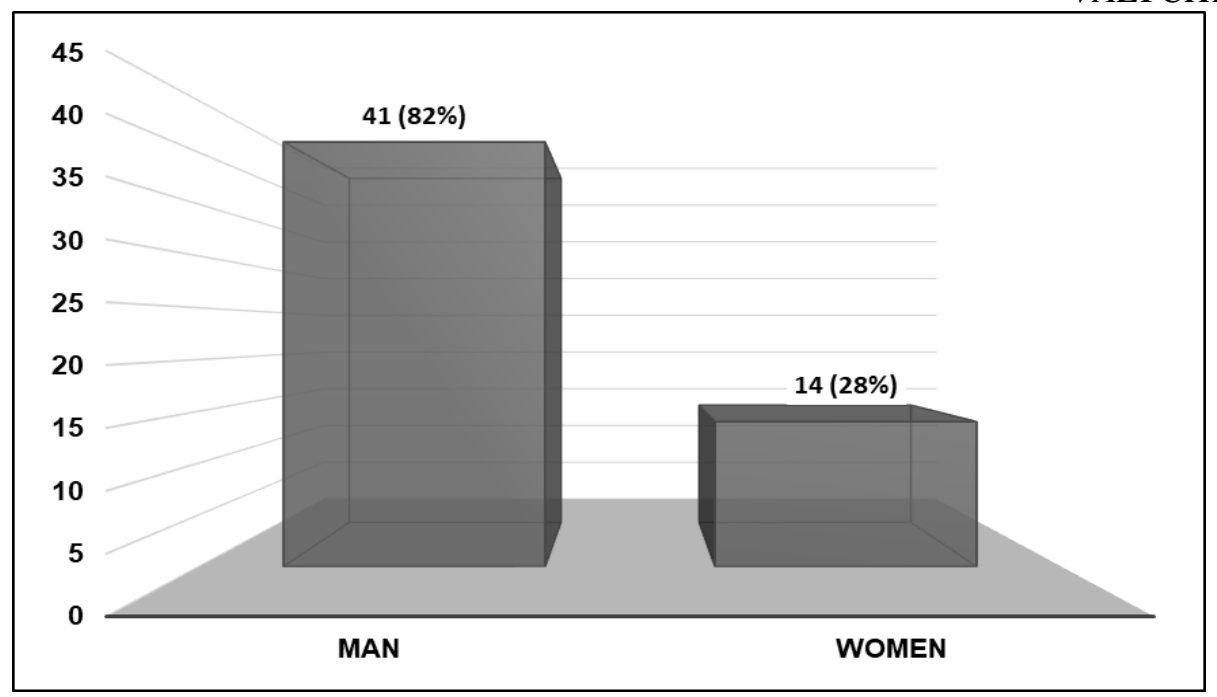

Figure 2. Distribution of bed bug-bitten athletes by sex

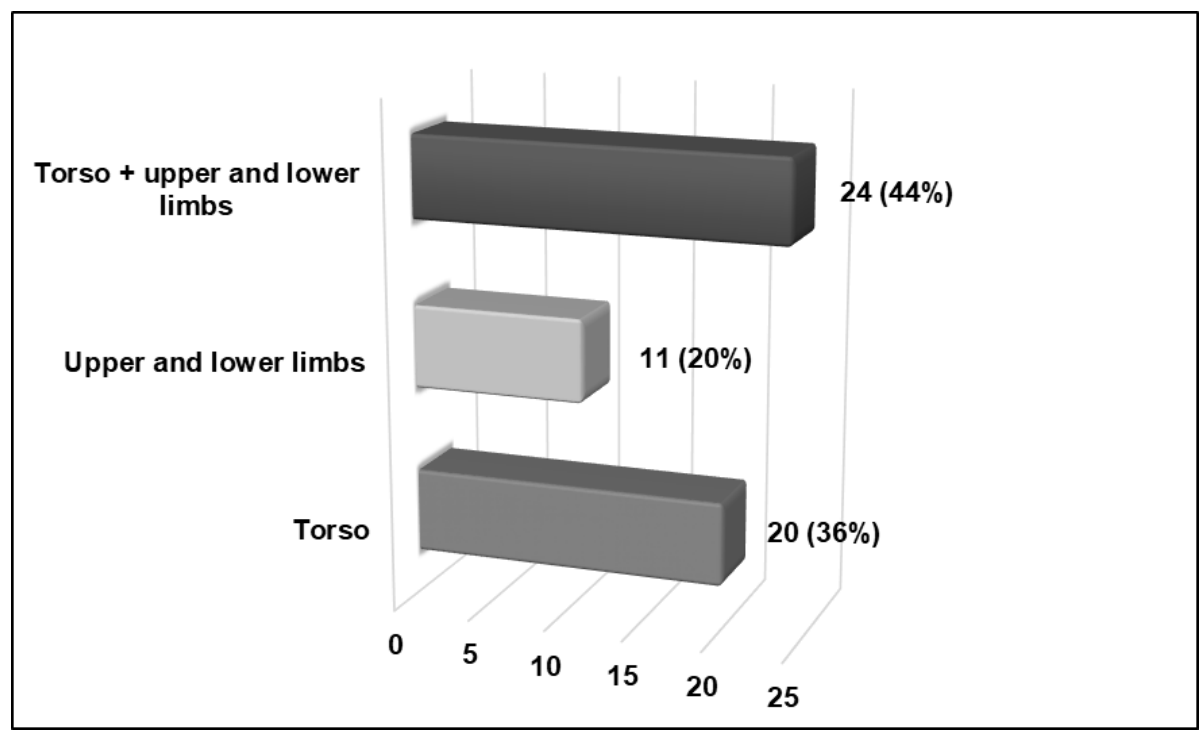

Figure 3. Localization of bed bug bites

The data presented in Figure 3 show that the percentage of bedbug bites is higher in men than in women because the majority of the studied contingent are men. Localization: 20 men have torso bites, 11 (4 women and 7 men) - have upper and lower limb bites and 24 (10 women and 14 men) have torso, upper and lower limbs affected.

In men, there is a higher concentration of bites in the torso. While in women, the bites are concentrated in the areas of the torso, upper and lower limbs. (Figure 4)

\section{TREATMENT}

Bed bugs are resistant and at lower temperatures have the ability to survive up to 2 years without food. It is extremely important that bed bugs be removed with the help of pest control professionals $(2,8,5)$.
To-date, there are no eradication plans in the fight against bedbugs $(4,5,9,10)$. Studies clearly show that bed bug management is a global health problem $(5,11-13)$. The frequency and prevalence of this ectoparasite worldwide are increasing endemically (4-6, 14). In practice, doctors cannot provide substantial treatment for people affected by bed bugs. Thus, the researchers investigated whether ivermectin (a relatively inexpensive and safe oral antiparasitic drug) was able to kill bed bugs after a blood meal from a host treated with the drug. The results of the study showed that an antiparasitic drug causes relatively high bed bug mortality (98\% for $260 \mathrm{ng} / \mathrm{mL}$ ivermectin and $0 \%$ for $0 \mathrm{ng} / \mathrm{mL}$ ivermectin after 13 days) $(14,15)$. Local corticosteroids could be used to treat pruritus. In severe cases, oral antihistamines are used. The most important thing is the destruction of bed bugs in the premises. (11) 


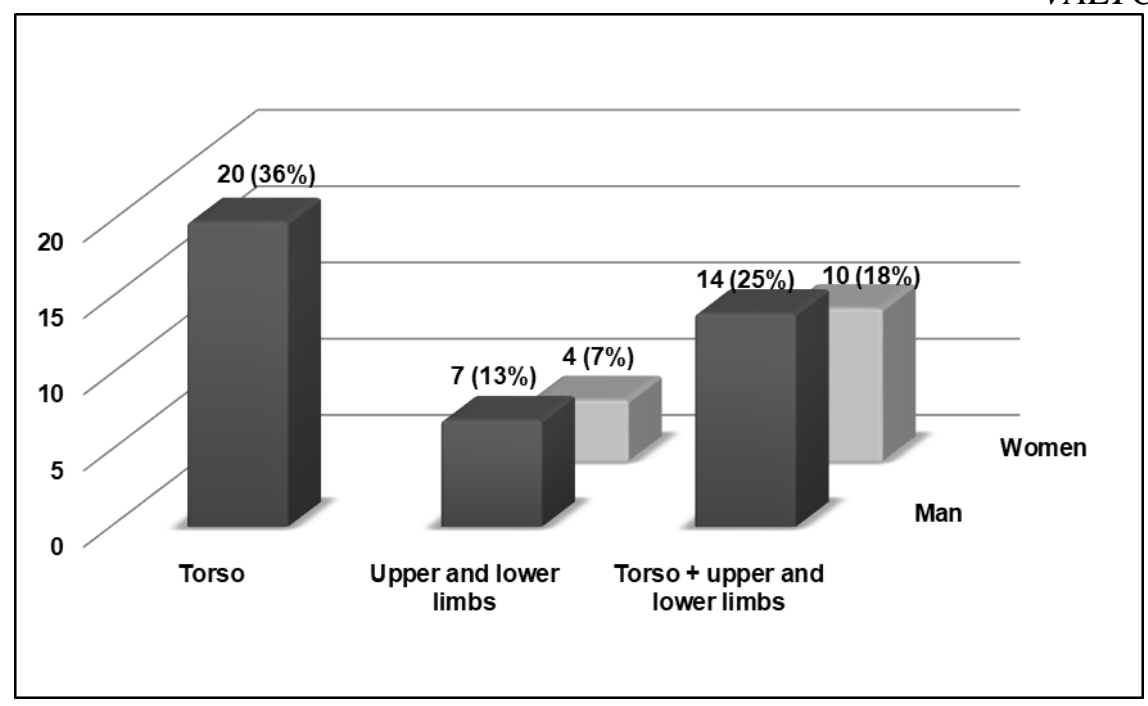

Figure 4. Localizations of male-female bites

In many countries around the world, specially trained dogs are also used to detect bed bugs $(9,16,17)$. Slovakia is the first country in Europe, which has specially trained certified bed bug search dogs. They can detect bed bugs by olfactory evidence corresponding to pheromones and fecal droplets of bed bugs. They can find whole nests of bed bugs in furniture and in the furnishing of an apartment. Currently, most of the populations of $C$. lectularius is resistant to organophosphates, pyrethroids and carbamates, which are most commonly used in experiments for killing them $(5,6,17)$. Their resistance to neonicotinoids has also been confirmed $(18,5)$. However, the organophosphates are banned in the EU, therefore it the use a mixture of deltamethrin and pyrethrum piperonylbutoxide for the control of $C$. lectularius is recommend $(6,14)$.

\section{CONCLUSION}

There is very little information on the frequency of bed bug bites in athletes in the available literature. This aroused our interest to present this material, which based on registered bed bug bites in athletes specializing in football. Bed bug bites and the accompanying skin irritation, which leads to unpleasant itching and impairs the quality of human life. Itching exacerbated when a person is under stress, during training or competition, and this prevents athletes from achieving good results. Itching is a natural reaction and can lead to temporary relief, but frequent scratching can further aggravate the itching. Scratching can damage the skin. Damaged skin is a gateway for infectious agents to enter.
Whenever an athlete has rash specialized medical attention should be sought.

To limit bedbug bites, while traveling in the country or abroad we can do the following:

1. Always check the hotel room for visible traces of bed bugs, the easiest way is to use a flashlight. If there are bed bugs or traces of them, we may ask to be moved to another hotel room. In the room, we have to check the mattress and the headboard of the bed, as well as the wardrobe in which we will leave our luggage.

2. In case of suspicion of bed bugs, we can protect the suitcase or bag we are traveling with by packing it in a plastic bag or sack, and the main idea for this is that bed bugs should not get into the suitcase or bag.

3 . In the hotel room do not use luggage shelves; do not keep suitcases and bags on the bed, near the bed or on the floor. These are the places where bed bug infestation most often occurs.

4. After returning from the trip, you can unpack your luggage directly in the bathroom. It is important to pay attention to the clothes you have traveled with. They can carry bedbugs and infect your home. Wash all clothes from your luggage in the washing machine at the highest possible temperature. The high temperatures from the dryer also destroy bed bugs. Normal laundry at low temperatures will not kill bed bugs.

5. Keep suitcases you travel away from the bedroom. After traveling, it is good to wash the suitcase or clean it with steam at a pressure of 120 degrees. Never store suitcases near your bed. 


\section{REFERENCE}

1. Valcthev V., Bacterial, viral and noninfectious dermatoses in athletes. NSA Press, monograph, p. 123, 2019.

2. Zlatkov N., Petranov E., Therapy of skin and sexually transmitted diseases. ARSO, Sofia, p. 650, 2000.

3. Manuelyan K., Drenovska K., Vasileva S., Bed bug bites (cimicosis). Dermatology and Venereology, 49(2): 64-68, 2011.

4. Kuhn C., Van der Pan., The worldwide expansion of bedbugs also constitutes a problem in Germany, Bundesgesundheitsblatt, 57(5): 524-530, 2014.

5. Leverkus M., Jochim R.C., Schäd S., Bröcker E.B., Andersen J.F., Valenzuela J.G., Trautmann A., Bullous allergic hypersensitivity to bed bug bites mediated by $\operatorname{IgE}$ against salivary nitrophorin. $J$ Invest Dermatol, 126(1):91-6, 2006.

6. Rupeš V., Bed bugs re-emerging in the Czech Republic. Bulletin of epidemiology and microbiology, 18(1): 17-18, 2009.

7. https://www.cdc.gov/parasites/bedbugs/faqs .html

8. Arayesh A., Wieczorek D., Kapp A., Raap U., Unclear pruritic and urticarial skin inflammation, Bed bug attack. Hautarzt. 64(3):187-9, 2013.

9. Thomas S, Wrobel MJ, Brown J., Bedbugs: A primer for the health-system pharmacist. Am J Health-System Pharmacy, 70(2): 12630, 2013.

10.Totková A., Ševčíková L., Argalášová L., Böhmer D., Repiská V., Malová J., Karkalík A., Totka A., Ectoparasite bed bug (cimex lectularius) has once again returned to human habitats also in Slovakia. Hygiena, 62(3):77-84, 2017.
VALTCHEV V., et al.

11.Doggett S.L., Dwyer D.E., Peñas P.F., Russell R.C., Bedbugs: clinical relevance and control options. Clin Microbiol, Rev. 25(1): 164-92, 2012.

12.Vaidyanathan R., Feldlaufer M.F., Bedbug Detection: Current Technologies and Future Directions. Am J Tropical Med Hygiene, 88(4): 619-625, 2013.

13.Wang C., Saltzmann K., Chin E., Bennett G.W., Gibb T., Characteristics of Cimex lectularius (Hemiptera: Cimicidae), infestation and dispersal in a high-rise apartment building. J Economic Entomol., 103(1): 172-177, 2010.

14.Zorrilla-Vaca A., Bedbugs and VectorBorne Diseases. Clin Infect Dis., 59(9): 1351-1352, 2014.

15. Sheele J.M., Anderson J.F., Tran T.D., Teng Y.A., Byers P.A., Ravi B.S., Sonenshine D.E., Ivermectin causes Cimex lectularius (bedbug) morbidity and mortality. J Emerg Med., 45(3): 433-14, 2013.

16.Pfiester M., Koehler P.G., Pereira R.M., Ability of bedbug - detecting canines to locate live bedbugs and viable bedbug eggs. J Economic Entomol., 101(4): 1389-1396, 2008.

17.Totkova A., Totka A., Sevcikova L., Argalasova L., Cibulkova A., Simko M., Problems with the bedbug (Cimex lectularius) in Slovakia. Annals of Agricultural and Environmental Medicine, 26 (3): 400-404, 2019.

18.Romero A., Anderson T.D., High Levels of Resistance in the Common Bedbug, Cimex lectularius (Hemiptera: Cimicidae), to Neonicotinoid Insecticides. J Med Entomol, 53(3): 727-731, 2016. 